\title{
Nano-Scale Observations of Tattoo Pigments in Skin by Atomic Force Microscopy
}

\author{
Colin A. Grant ${ }^{a}$ Peter C. Twigg ${ }^{a}$ Desmond J. Tobin ${ }^{b}$ \\ ${ }^{a}$ Department of Medical Engineering, Faculty of Engineering and Informatics, ${ }^{b}$ Centre for Skin Sciences, \\ Faculty of Life Sciences, University of Bradford, Bradford, UK
}

\begin{abstract}
In this study, we have shown how particles in carbon black tattoo ink accumulate in the human skin dermis using fine-resolution atomic force microscopy, with which a single ink particle in the collagenous network can be imaged. This information further demonstrates that tattoo inks are nano-particles. Further, we have deposited a commercially available tattoo ink on a glass slide and calculated a range of volumes for single ink particles.
\end{abstract}

๑) 2015 S. Karger AG, Basel

\section{Introduction}

The skin, which is the body's largest organ, is positioned at the interface between the external environment and internal anatomical structures, and as such, it provides a barrier against a range of environmental hazards [1]. Recent trends in the 21st century have shown an increase in the number of people with one or more tattoos or body art, some covering large regions of the skin's surface. Tattoo inks consist of a solid pigment dispersed in an aqueous medium that acts as a carrier and is usually a combination of an alcohol and water. Neither tattoo ink manufacturers nor tattoo artists who concoct their own ink have revealed the components or their precise proportions in their inks. Solid pigments for black ink are usually made from carbon black; however, a study of 58 inks of 6 different colours has shown that these pigments are predominantly less than $100 \mathrm{~nm}$ in diameter [2]. Nano-particles (defined as particles under $100 \mathrm{~nm}$ in size) are generally found to be more chemically reactive due to their high surface-to-volume ratios and are potentially cytotoxic because they may be able to pass through cell membranes.

For many centuries, microscopes have been invaluable instruments used by scientists, especially in the biological sciences. However, the resolution of light microscopes is limited due to diffraction, and electron microscopes require vacuum conditions. The atomic force microscope (AFM) was first created in the 1980s, invigorating the microscopy field [3]. The AFM possesses a long silicon (or silicon nitride) cantilever/probe assembly (fig. 1a) with a sharp probe on the underside of the cantilever with a half-cone angle of approximately $36^{\circ}$, and it 


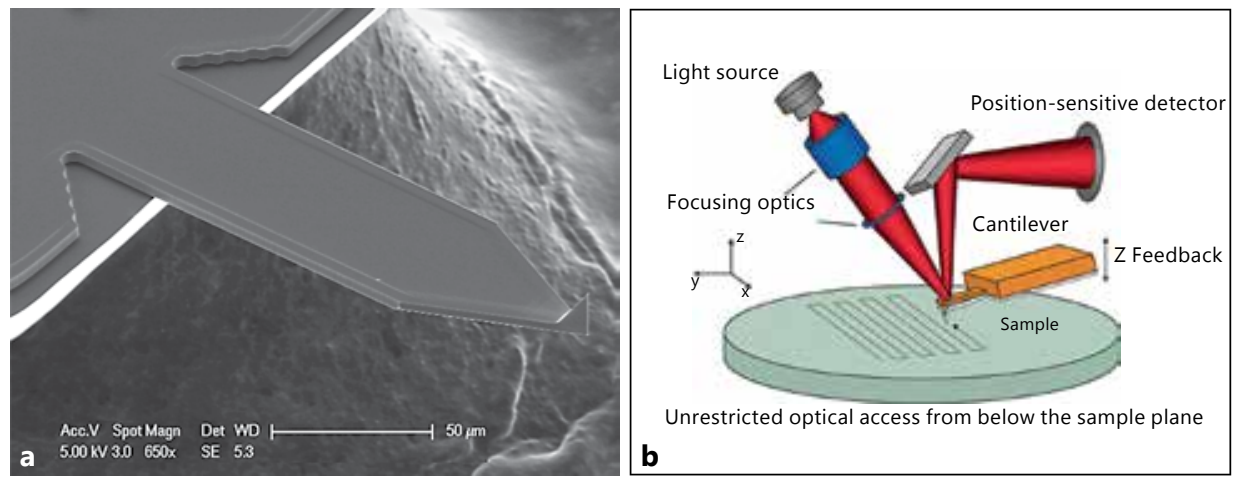

Fig. 1. a Scanning electron microscopy image of AFM silicon cantilever OMCL-AC160TS-R3 (diagram courtesy of Olympus Corporation, Japan). b Schematic of mechanism of surface scanning with an AFM (diagram courtesy of Oxford Instruments Asylum Research, USA).

typically has a spherical radius of approximately $10-20 \mathrm{~nm}$.

Intricate details of how an AFM works can be found elsewhere [4]. In brief, a laser is aligned to the back of the cantilever/probe assembly, which itself is angled downward at approximately $12^{\circ}$ (fig. 1b). Then, the cantilever/probe assembly is oscillated at its resonant frequency by a piezoelectric motor. The oscillating probe is brought into intermittent contact with a sample surface and is scanned over an area of interest, from a few nanometres up to a maximum of $100 \mu \mathrm{m}$. Changes in oscillation amplitude due to surface topography deflect the laser beam and bring about a change in signal at the photo-detector. This is a feedback channel that ultimately changes the vertical $(Z)$ distance between the tip and sample, resulting in a surface image with contrast based on height, amplitude (error) or phase lag, which can reveal structures not present in the height image.

Advanced AFM instruments using low temperatures and high vacuum conditions have been used to image atoms [5] and even molecular bonds $[6,7]$. However, an AFM can work under both air and aqueous conditions, and with the appropriate equipment, it can run at a wide range of temperatures of up to $\sim 300^{\circ} \mathrm{C}$. This makes it ide- al for investigations of biological or anatomical samples under representative physiological conditions. Further, the cantilever/probe assembly can be used both as a tool for surface visualisation and as an indentation device to extract material properties from a surface of interest. For example, it has been recently demonstrated that the elasticity of a single collagen fibril is reduced by 3 orders of magnitude simply by hydrating a sample [8] and that collagen properties can be tuned by varying the aqueous media [9]. The discrete, periodic banding patterns of collagen fibrils have different static and dynamic mechanical properties [10]. Finally, surface images of skin scar tissue have shown a high degree of orientation of collagen fibrils as a result of wound healing with variations in the mechanical properties of the tissue in comparison with healthy skin [11]. In this study, for the first time, we utilise this microscopy technique to evaluate tattooed skin samples.

\section{Methods}

\section{Cryo-Sectioning Tissue}

A sample of tattooed skin was obtained from the forearm of a 62-year-old male. Full consent was obtained from the ethics committee of the clinic and the 
Fig. 2. Top-down optical microscope image of an AFM cantilever in the proximity of tattoo ink agglomerates in a dermis skin section. Scale bar, $200 \mu \mathrm{m}$.
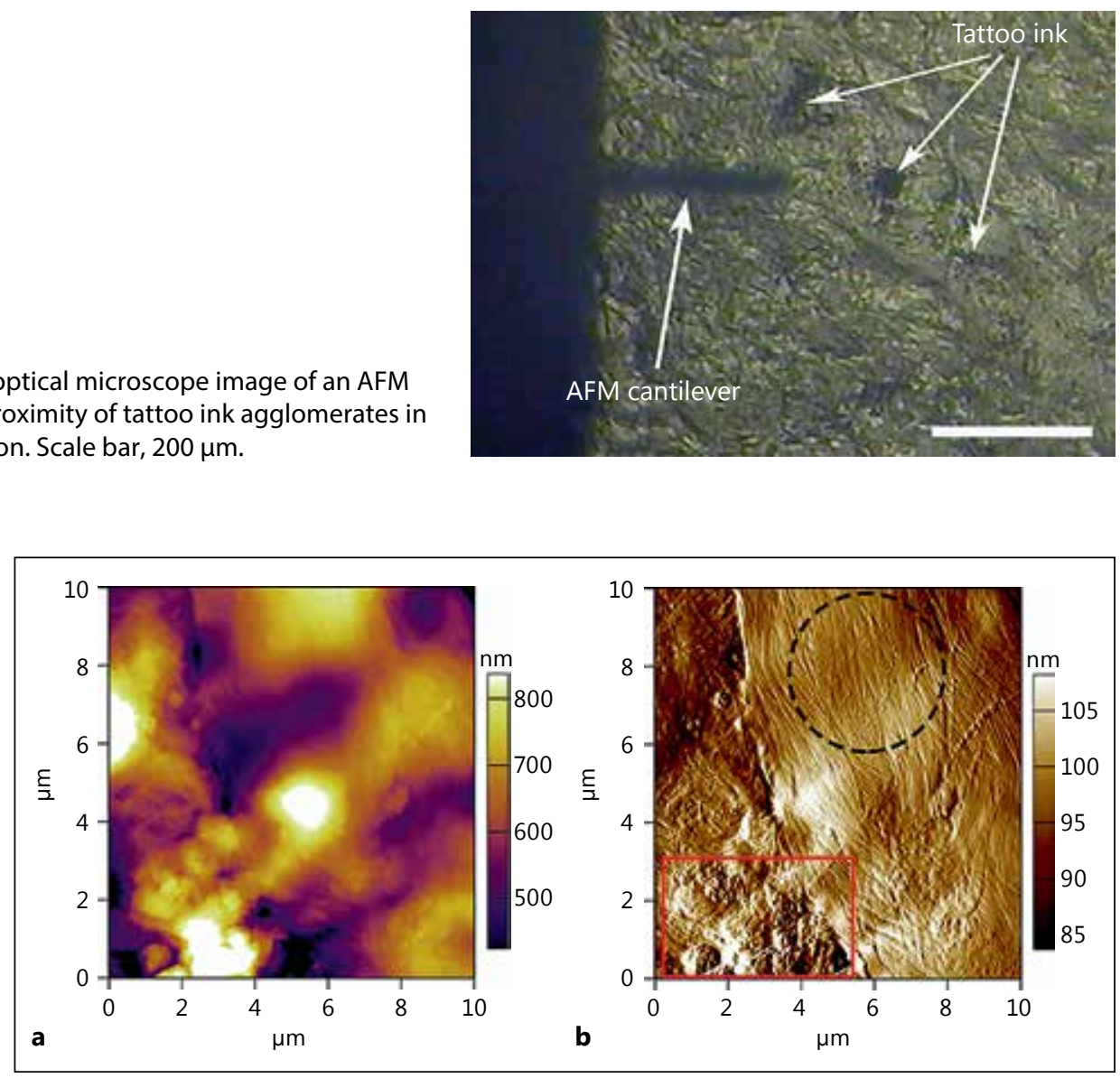

Fig. 3. A 10- $\mu \mathrm{m}$ AFM image of a (a) height image and a corresponding (b) amplitude image.

university for the use of this tissue. The tattooed skin sample was collected in buffered saline and stored upon arrival at the laboratory in a $-80^{\circ} \mathrm{C}$ freezer. The skin sample was placed in a cryostat (Leica CM1860) and adhered to a metal chuck using an optimum cutting temperature embedding compound (Agar Scientific, Essex, England). Thin sections of dermal skin (5 $\mu \mathrm{m})$ were collected onto poly-lysine-coated microscope slides and stored at $-80^{\circ} \mathrm{C}$ until analysis.

\section{Atomic Force Microscopy}

The microscope slides containing the cryo-sectioned tissue sample were placed on the sample stage of an MFP-3D AFM (Asylum Research, Santa Barbara, Calif., USA) and imaged in air in intermittent contact mode using Olympus AC160 silicon probes $(\mathrm{k} \sim 40$ $\mathrm{N} / \mathrm{m}$, tip radius $\sim 10 \mathrm{~nm}$ ). A suitable area of interest at the periphery of the tattoo ink aggregates was located using an AFM optical camera $(10 \times)$ before engaging on the surface and imaging (fig. 2). All images were processed using AFM software based on IGOR Pro platform (Wavemetrics, USA).

A 10- $\mu \mathrm{m}$ AFM scan image is shown in figure 3, in which (a) is the height image, and (b) is the amplitude or error image. The skin in this region of interest is clearly undulating with a large vertical data scale, which causes the nano-particles to be very difficult to discern.

However, from the amplitude image, a number of observations can be made. The right-hand side of the 


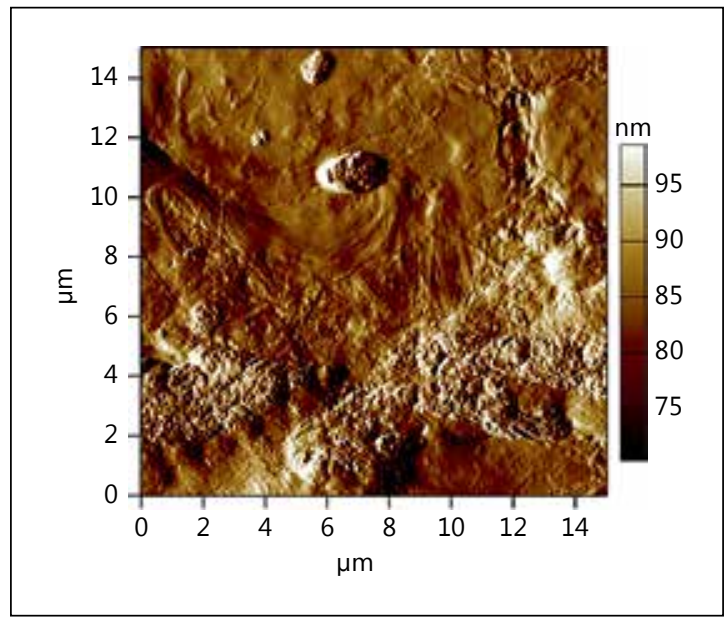

Fig. 4. A $15-\mu \mathrm{m}$ amplitude image showing the greater density of ink pigment aggregates within the collagenous network.

amplitude image shows the strong orientation of the collagen fibrils, which is known to occur following wound healing or trauma [11] (in this case, receiving a tattoo). There are a number of small individual ink particles in the region of the highly oriented collagen fibrils, which are shown within the dashed circle in the amplitude image (fig. 3b) and are not visible in the corresponding height image (fig. 3a). Further, at the bottom left of figure $3 \mathrm{~b}$ in the red rectangle, there are 'clumps' of aggregated ink particles that can be seen better in the amplitude image compared with the height image.

With a larger field of view, as shown in figure 4, clumps of tattoo pigment aggregates can be seen together with a large accumulation of pigment that dominates the lower half of the $15-\mu \mathrm{m}$ image. It should be noted that because the tattooed dermis was cryo-sectioned at $5 \mu \mathrm{m}$, the tattoo pigment clusters are located at the sectioned tissue surface as well as sub-surface. The skin surface appears unscathed by the cryo-sectioning; otherwise, there would be shear-induced damage to the tissue.

The AFM has an impressive resolution capability in the $\mathrm{z}$ (vertical) direction down to the Angstrom level. Figure 5 a shows a $2-\mu \mathrm{m}$ AFM amplitude image of clusters of tattoo ink pigment particles dispersed within the collagen network. Figure 5b reveals a single ink particle with a diameter of $30 \mathrm{~nm}$, as measured

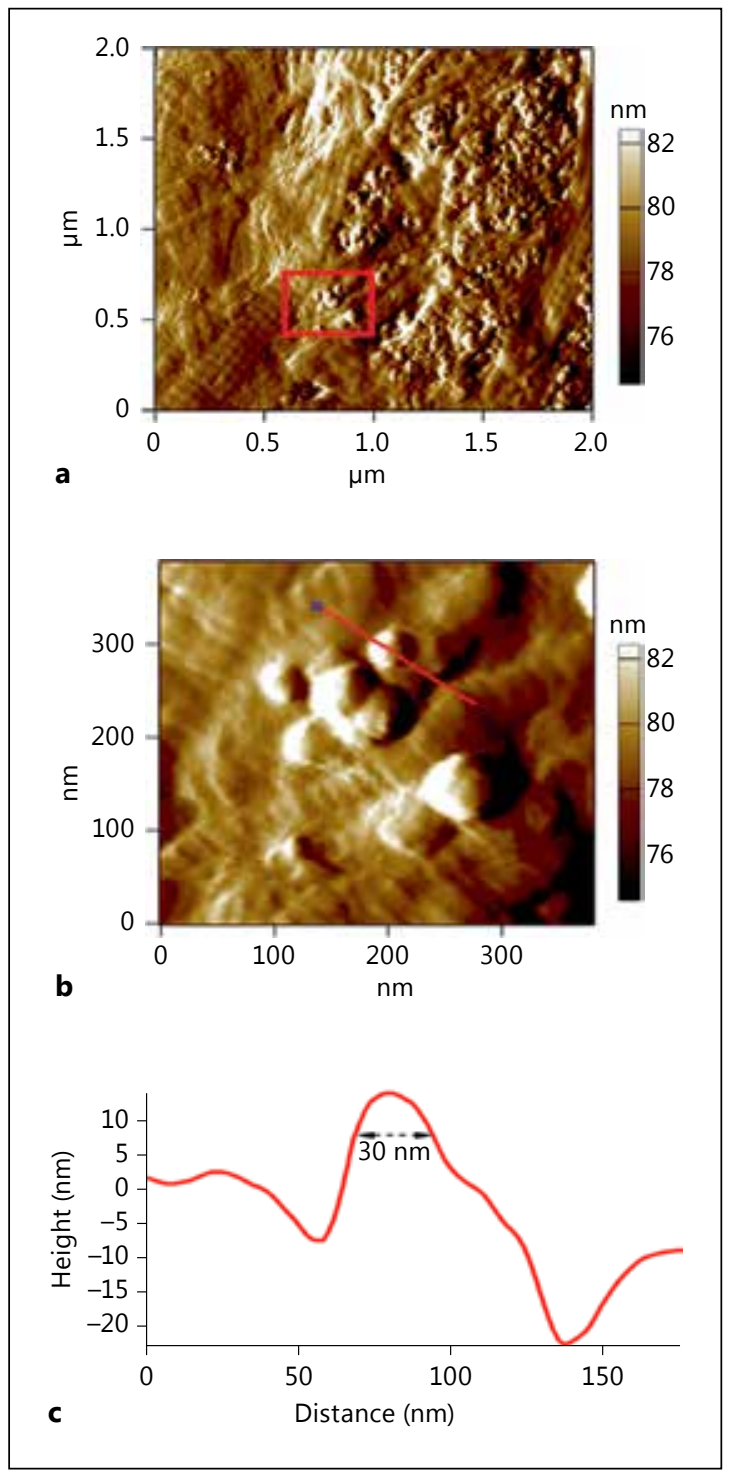

Fig. 5. a A 2- $\mu \mathrm{m}$ amplitude image showing thinly dispersed ink pigments. b A 400-nm scan region from the red square, resolving a primary particle. c A line section over a primary pigment particle, showing the profile of the surface.

from a line profile (fig. 5c) within a cluster of ink particles. Because the use of an AFM involves surface metrology, the particle shown is likely to be embedded in the collagen network and may be larger than calculated. Unfortunately, the age/duration of the 

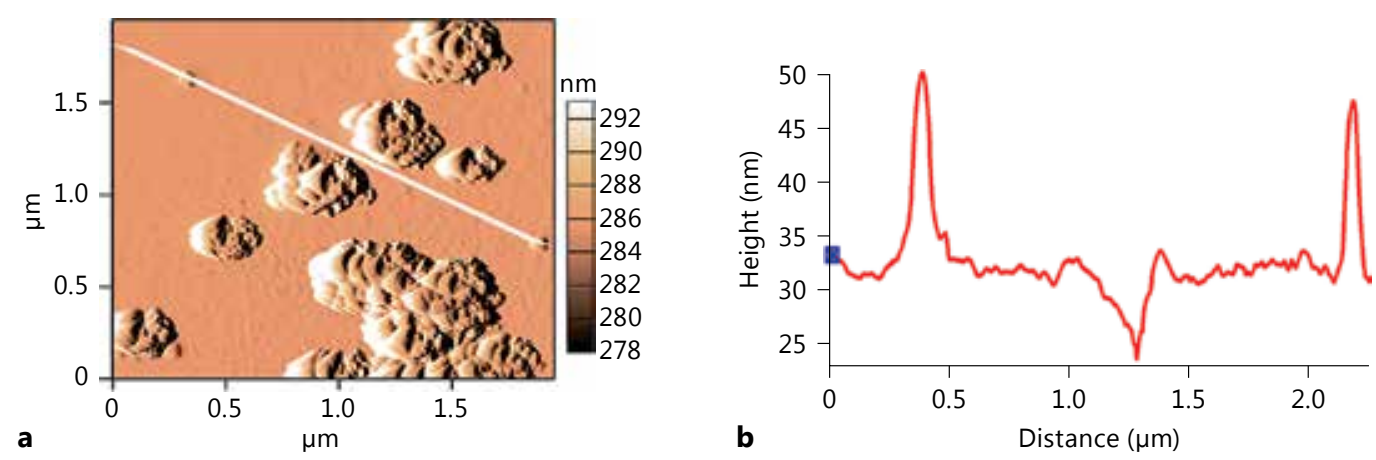

Fig. 6. a A 2- $\mu \mathrm{m}$ amplitude scan of tattoo ink on a glass slide. b A line section along the black, dashed line linking particle 1 and particle 2 .

tattoo and the identity of the ink(s) used on the arm of the 62-year-old subject are not known, although the surface colour was monochrome (blue/black). The ink pigments in the skin are likely to have diffused/repositioned over time since the original tattoo event because it is known that deposited ink pigments are typically not stationary and are subjected to the cell-mediated non-self immune response of the body.

To demonstrate the validity of the AFM images of the ink pigments deposited in the dermal collagenous networks, we imaged a commercially available tattoo ink (Scream Black, Tattoo World UK) at the primary particle level as a control. The tattoo ink was diluted $(1: 1,000)$, and a $100-\mu l$ droplet was placed on a polylysine-coated glass slide. Following 10 minutes of deposition time, the ink was washed off of the surface and gently dried with a stream of nitrogen and then placed under an AFM. The resulting AFM scan of the dispersed ink particles is shown in figure 6a. Despite being a different black tattoo ink source, there was a striking similarity in the accumulation of the pigments on the skin and the glass substrate (c.f. fig. 3b, 4, 5a, b with fig. 6).

A line section over two individual particles on the glass surface is shown in figure $6 \mathrm{~b}$, in which the heights of the particles are 17 and $16 \mathrm{~nm}$, respectively, and the widths are 68 and $42 \mathrm{~nm}$, respectively. Assuming that these two single pigments are representative and have near-circular geometries, these dimensions indicate a volume of ink pigment particles of between 1,385 and
$3,631 \mathrm{~nm}^{3}$. Putting these values into context, a spherical nano-particle of $100 \mathrm{~nm}$ in diameter would have a volume of $523,599 \mathrm{~nm}^{3}$. We hypothesise that single, isolated, primary nano-particles in the dermis could well be the origin of allergic responses due to the higher reactivity of smaller-sized nano-particles compared with the agglomerated particles found in tattoo skin. Nanomaterials are known to exhibit different properties from their bulk counterparts; the smaller the particle, the larger the fraction of atoms at the surface and the larger the binding energy per atom, resulting in surface atoms that are less stable (i.e. more reactive) than bulk atoms [12].

\section{Conclusion}

This preliminary study has shown that the AFM can resolve ink nano-particles from sections of tattooed human skin. The size of the individual tattoo ink particle in the dermal network, as resolved by an AFM, was approximately $30 \mathrm{~nm}$. The clusters of ink attached to the glass substrate in the control experiment showed a striking similarity to the agglomerated ink pigments found in tattooed skin. With ink pigments on glass, line profiles can be used to measure surface area and height, revealing the volume of a single ink pigment. 


\section{References}

1 Tobin DJ: Biochemistry of human skinour brain on the outside. Chem Soc Rev 2006;35:52-67.

-2 Høgsberg T, Loeschner K, Löf D, Serup $\mathrm{J}$ : Tattoo inks in general usage contain nanoparticles. Br J Dermatol 2011;165: 1210-1218.

3 Binnig G, Quate CF, Gerber C: Atomic force microscope. Phys Rev Lett 1986; 56:930-933.

4 Santos NC, Castanho MARB: An overview of the biophysical applications of atomic force microscopy. Biophys Chem 2004;107:133-149.

5 Giessibl FJ: AFM's path to atomic resolution. Materials Today 2005;8:32-41.
6 Gross L, Mohn F, Moll N, Liljeroth P, Meyer G: The chemical structure of a molecule resolved by atomic force microscopy. Science 2009;325:1110-1114.

7 Gross L, Mohn F, Moll N, Schuler B, Criado A, Guitián E, Peña D, Gourdon A, Meyer G: Bond-order discrimination by atomic force microscopy. Science 2012;337:1326-1329.

8 Grant CA, Brockwell DJ, Radford SE, Thomson NH: Effects of hydration on the mechanical response of individual collagen fibrils. Appl Phys Lett 2008;92: 3902-3904.
9 Grant CA, Brockwell DJ, Radford SE, Thomson NH: Tuning the elastic modulus of hydrated collagen fibrils. Biophys J 2009;97:2985-2992.

10 Grant CA, Phillips MA, Thomson NH: Dynamic mechanical analysis of collagen fibrils at the nanoscale. J Mech Behav Biomed Mater 2012;5:165-170.

11 Grant CA, Twigg PC, Tobin DJ: Static and dynamic nanomechanical properties of human skin tissue using atomic force microscopy: effect of scarring in the upper dermis. Acta Biomater 2012;8: 4123-4129.

12 Roduner E: Size matters: why nanomaterials are different. Chem Soc Rev 2006; 35:583-592. 